\title{
Single-picture reconstruction and rendering of trees for plausible vegetation synthesis
}

\author{
Anonymous
}

\begin{abstract}
State-of-the-art approaches for tree reconstruction either put limiting constraints on the input side (requiring multiple photographs, a scanned point cloud or intensive user input) or provide a representation only suitable for front views of the tree. In this paper we present a complete pipeline for synthesizing and rendering detailed trees from a single photograph with minimal user effort. Since the overall shape and appearance of each tree is recovered from a single photograph of the tree crown, artists can benefit from georeferenced images to populate landscapes with native tree species. A key element of our approach is a compact representation of dense tree crowns through a radial distance map. Our first contribution is an automatic algorithm for generating such representations from a single exemplar image of a tree. We create a rough estimate of the crown shape by solving a thin-plate energy minimization problem, and then add detail through a simplified shape-from-shading approach. The use of seamless texture synthesis results in an image-based representation that can be rendered from arbitrary view directions at different levels of detail. Distant trees benefit from an output-sensitive algorithm inspired on relief mapping. For close-up trees we use a billboard cloud where leaflets are distributed inside the crown shape through a space colonization algorithm. In both cases our representation ensures efficient preservation of the crown shape. Major benefits of our approach include: it recovers the overall shape from a single tree image, involves no tree modeling knowledge and minimal authoring effort, and the associated image-based representation is easy to compress and thus suitable for network streaming.
\end{abstract}

Keywords:

Tree reconstruction, Tree rendering, Vegetation synthesis

\section{Introduction}

Publicly available Digital Terrain Models (DTM) and associ3 ated aerial images have opened new possibilities for using real 4 scenarios in video games and entertainment applications. Since 5 vegetation is poorly represented in current DTMs, artists of${ }_{6}$ ten populate the terrain with realistic tree models to support 7 close-up views of the scenario (as required e.g. in racing video 8 games). Unlike imaginary scenarios, where tree appearance can ${ }_{9}$ be left to artists' criteria, a substantial amount of modelling ef10 fort is required to create realistic tree models matching the local ${ }_{11}$ species of a given forest area (e.g. there are more than forty va12 rieties of pine trees, each one native to specific regions around ${ }_{13}$ the world). This last requirement favors tree reconstruction over ${ }_{14}$ modeling, as the former better reproduces existing tree varieties 15 and, in most cases, reduces authoring efforts.

${ }_{16}$ In this paper we present a pipeline to reconstruct and ren${ }_{17}$ der plausible trees. Unlike most existing approaches for tree ${ }_{18}$ reconstruction, which require either a point cloud or multiple ${ }_{19}$ photographs of each species, we extract the overall shape and 20 appearance of the tree from a single photograph of the tree (Fig${ }_{21}$ ure 1). This way we can benefit from georeferenced images ${ }_{22}$ from the web to synthesize trees for a particular location. We ${ }_{23}$ extract three basic pieces of information from the tree photo${ }_{24}$ graph. The overall shape is extracted from the crown silhouette ${ }_{25}$ via a bilaplacian filter. Crown color from the photo is used to ${ }_{26}$ synthesize a seamless, $360^{\circ}$ color texture for the whole crown.
${ }_{27}$ Crown luminance is used to compute a relief texture through 28 a shape-from-shading method, providing mid-frequency and 29 high-frequency details on the crown shape.

Our method starts with a picture of a tree crown, together ${ }_{31}$ with a foreground/background segmentation of its foliage (Fig${ }_{32}$ ure 1). From this picture, we generate two outputs: a color map 3 representing the appearance of the foliage, and a radial distance ${ }_{34}$ map (RDM) storing the maximum distance from the crown cen${ }_{35}$ ter to the crown surface, i.e. for each unit vector we store the ${ }_{36}$ radius from the center to the outermost silhouette. Both textures ${ }_{37}$ are stored as cube maps and used at render time. The genera38 tion of the RDM requires creating a temporary mesh represent39 ing the overall shape of the crown, which is then combined with 40 an estimation of the crown relief extracted from the color map ${ }_{41}$ using a shape-from-shading approach.

Once the RDM has been generated, the resulting crown vol${ }_{43}$ ume can be used as input for a Space Colonization algorithm [1] ${ }_{44}$ to generate a branching structure at the desired detail level. ${ }_{45}$ Since we assume the crown is dense enough, we only need to ${ }_{46}$ reconstruct and display the main branches. This contrasts to ${ }_{47}$ previous approaches, which reconstruct a complete underlying ${ }_{48}$ branching structure beforehand and then add the leaves.

Our representation allows for efficient rendering of thou${ }_{50}$ sands of tree instances. Distant trees are rendered with an ${ }_{51}$ output-sensitive relief mapping approach. For close-up views, ${ }_{52}$ we switch to a representation using textured billboards clipped 


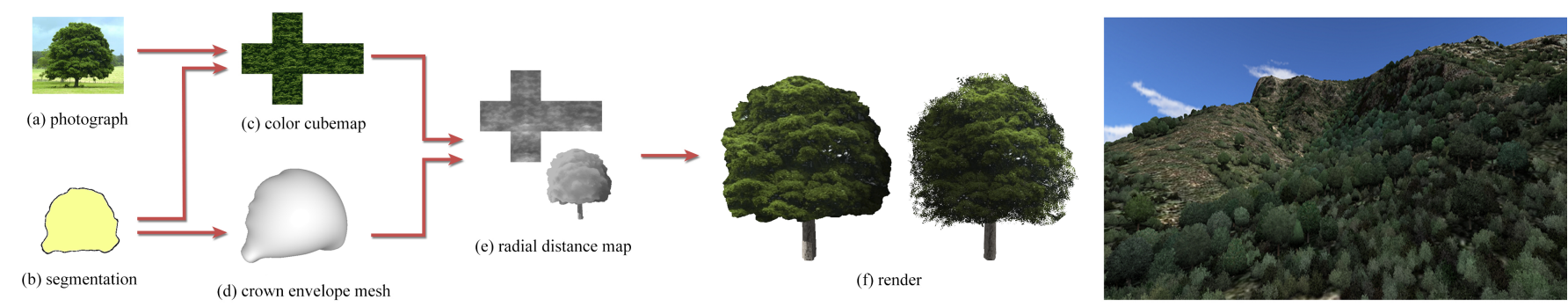

Figure 1: We present an algorithm to reconstruct trees from a single photograph. The resulting models can be used to synthesize plausible vegetation on top of arbitrary terrains. Our approach can benefit from georeferenced images from the web to populate landscapes with native tree species.

53 against the crown volume.

${ }_{54}$ The rest of the paper is organized as follows. Section 2 re${ }_{55}$ views previous work on tree modeling, reconstruction and ren${ }_{56}$ dering. Section 3 describes the reconstruction of the tree, and ${ }_{57}$ Section 4 is devoted to tree rendering. Section 5 discusses our ${ }_{58}$ results with a variety of tree species. Concluding remarks are 59 provided in Section 6.

\section{2. Previous work}

61 Tree modeling The lack of the hardware needed to capture ${ }_{62}$ and process real tree models directed early efforts towards tree ${ }_{63}$ modeling. The first contributions focused on the effect of cer${ }_{64}$ tain parameters (such as branching angle and branch length) on ${ }_{65}$ tree shapes [2]. These findings were applied to recursive algo${ }_{66}$ rithms which produced the first tree structures resembling their ${ }_{67}$ real counterparts [3]. The parameters of these algorithms al${ }_{68}$ lowed the user to alter the generated model in real-time, making ${ }_{69}$ it possible to examine the model space interactively [4]. One of 70 the main drawbacks of such techniques is the large number of ${ }_{71}$ parameters required to specify a particular model [5]. It is pos72 sible though to obtain values for these parameters from a given ${ }_{73}$ tree model that can then be used to generate similar ones [6]. ${ }_{74}$ All these methods allow generating the branching skeleton of a 75 tree but they transform the resulting hierarchy into a 3D model ${ }_{76}$ using simple modeling algorithms. Bloomenthal et al. [7] ex77 amined the transformation process proposing techniques to rep78 resent the trunk, branches, and bark of a tree more faithfully.

79 Another set of techniques were introduced by Linden80 mayer [8] that, exploiting the capabilities of formal languages, ${ }_{81}$ managed to imitate plant development. L-systems have been 82 widely used for modeling all types of plants and have been ex${ }_{83}$ tended to support most of its peculiarities. These extensions ${ }_{84}$ include the integration of production rules to represent the de${ }_{85}$ velopment of the plant over time [9], the interaction with the ${ }_{86}$ environment [10], the expression of plant attributes based on ${ }_{87}$ their spatial location [11], and many others [12]. Despite the ${ }_{88}$ difficulty of predicting the outcome produced by this type of ${ }_{89}$ grammars, it is possible to control the process and produce the ${ }_{90}$ desired output [13]. Lintermann et al. [14] introduced a set of ${ }_{91}$ algorithms that encapsulated components and structures associ${ }_{92}$ ated with key parts present in most plants. These components ${ }_{93}$ could be combined using software similar to most geometric
${ }_{94}$ modelers, thus resulting in a more intuitive way of modeling 95 plants.

96 One recent strategy is based on the observation of the factors ${ }_{97}$ that influence the final shape of a tree. In particular, compe${ }_{98}$ tition for resources (sunlight, space) by different branches of 99 a tree seems to be critical for the general shape of temperate100 climate trees. The space colonization algorithm [1] uses this ${ }_{101}$ fact to establish a set of attractors in the volume defined by the 102 tree's foliage, which is iteratively conquered by the branches as 103 they occupy the available space. Palubicki et al. [15] extended 104 this algorithm using a signaling mechanism to mimic different 105 types of growth. It is also possible to provide the overall crown 106 shape using a gesture based system that guides the resulting 107 tree's growth [16], reducing modeling time while maintaining ${ }_{108}$ the artist's ability to obtain the desired result.

Other factors such as wind and surrounding space also influ110 ence a tree's growth. Pirk et al. [17] presented a technique that 111 made it possible for content creators to change a tree's position 112 inside a scene and see its shape adapt to changes in light distri${ }_{113}$ bution and the occupation of surrounding space. In [18] it was 114 extended to include the effect of wind.

115 Tree reconstruction Only recently interest has shifted to 116 the reconstruction of trees, either from multiple photographs or ${ }_{117}$ point clouds obtained from laser scanners. Shlyakhter et al. [19] ${ }_{118}$ proposed an algorithm that took several photographs as input ${ }_{119}$ and used the segmentation of the crown to construct the tree's ${ }_{120}$ visual hull. The resulting shape was filled with a plausible tree ${ }_{121}$ skeleton that they augmented using L-systems. To improve the 122 segmentation stage Reche et al. [20] applied an image matting ${ }_{123}$ algorithm, combining the results to generate an opacity vol124 ume. The reconstructed trees were rendered view-dependently ${ }_{125}$ attaching billboards to opaque cells. Another possible strategy ${ }_{126}$ Would be using the volume to generate the initial positions of ${ }_{127}$ a 3D flow simulation, where the trunk and first-order branches 128 were used as attractors [21].

Some techniques take a hybrid approach. In order to gener130 ate the model, they transform their input image collection into ${ }_{131}$ a point cloud using a structure-from-motion algorithm. Quan ${ }_{132}$ et al. [22] used the generated point cloud to extract and seg${ }_{133}$ ment individual leaves allowing the user to model the branches. ${ }_{134}$ On the other hand, Tan et al. [23] synthesized hidden branches ${ }_{135}$ from visible ones with texture-based synthesis algorithms and ${ }_{136}$ then added the crown instantiating leaf clusters identified in the ${ }_{137}$ point cloud. Also starting from a point cloud (in addition to an 
${ }_{138}$ example of a mesh leaf) the technique by Bradley et al. [24] is ${ }_{139}$ capable of generating plausible foliage configurations. In order ${ }_{140}$ to do this, it fits the exemplar leaf to the point cloud, extracting a ${ }_{141}$ statistical model of the shape, appearance, and transformations 142 between neighbors.

${ }_{143}$ Scanned point sets have also been used to extract tree ge${ }_{144}$ ometry. Xu et al. [25] classified points into two types: leaves 145 and branches. Their method builds a graph connecting neigh${ }_{146}$ bors that is used to produce a skeleton, extends it into the tree ${ }_{147}$ crown and transforms it into a mesh. Other approaches refine 148 the initial branch-structure graph according to some optimiza${ }_{149}$ tion criteria [26]. It is also possible to decompose a tree's crown 150 into geometry structures called lobe-textures [27].

${ }_{151}$ A 3D tree model may also be generated from a 2D sketch ${ }_{152}$ assuming that trees spread their branches far apart from each ${ }_{153}$ other [28]. Such system also allows users to apply gesture154 based editing operations and manually generate trees from ${ }_{155}$ given examples. Wither et al. [29] made use of successive sil156 houettes sketched at different zoom levels to create a 3D tree.

${ }_{157}$ Most reconstruction methods that take photographs as input 158 need either several images to work properly or significant user 159 interaction. The technique proposed in [30] is a notable ex${ }_{160}$ ception and our direct competitor, at it is able to extract the ${ }_{161}$ branching structure from a single image of the tree. The user ${ }_{162}$ draws one stroke in the photograph to identify the crown, and ${ }_{163}$ another one (or sometimes more) for the branches. The crown 164 is segmented and the visible branches are converted to 3D us165 ing the approach proposed in [28]. The initial skeleton is ex${ }_{166}$ tended into the crown by iteratively substituting an existing ${ }_{167}$ branch by a subtree from a database. Unlike [30], our image${ }_{168}$ based representation is much more compact and thus suitable ${ }_{169}$ for efficient streaming and rendering of forest scenes. Our ap170 proach also supports arbitrary camera rotations around the tree, 171 whereas [30] is intended for still renderings of front and side 172 views of the tree due to the direct use of the input image as tex173 ture. On the other hand, we focus on the reconstruction of tree 174 foliage instead of the branching structure that, for dense trees, 175 would be hardly visible. As a downside, we cannot reconstruct 176 sparse trees, which are correctly handled by [30].

Tree rendering Plants and ecosystems are extremely com${ }_{178}$ plex objects and their interactive rendering is still a challeng179 ing problem. Research focused on rendering large forests has ${ }_{180}$ developed multi-scale systems [31] that use several representa${ }_{181}$ tions of each tree. One option is to use particle systems [32] as 182 they can represent irregular 3D volumetric structures with ease. 235 ${ }_{183}$ One could also substitute a plant model by a set of points and ${ }_{230}$ 184 line primitives [33]. Such a model allows to smoothly reduce 185 detail by rendering a progressively smaller subset of those basic 186 primitives. Then, dividing the scene hierarchically, we can ap187 ply different ratios of reduction to each region. Gilet et al. [34] ${ }_{188}$ proposed a technique that uses point clouds to represent vege189 tation. Several instances of one or more blocks of vegetation 190 compose a landscape and each block is rendered using either its 191 original polygonal model or a decimated point cloud generated 192 from it. Decaudin and Neyret [35] covered the ground of the 193 forest they wanted to display with a volumetric texture that was 194 rendered using texture slicing. Andujar et al. [36] synthesize
195 procedural vegetation visually compatible with low-resolucion 196 aerial images by using a rotationally-symmetric crown model 197 which is rendered by modulating the aerial color with proto198 type leaf textures. Compared to [36], our approach automat199 ically generates a faithful representation of the crown volume 200 and appearance from a photograph of the tree crown, resulting 201 in clearly distinguishable tree specimens.

More recently this problem has been approached from a 203 simplification viewpoint, the difficulty being that vegetation is ${ }_{204}$ composed of a large number of already very simple elements. ${ }_{205}$ Cook et al. [37] addressed it by rendering them using a ran206 domly selected subset of elements statistically altered to pre207 serve the overall appearance. Then Neubert et al. [38] improved 208 upon the previous technique by defining a priority based prun209 ing that preserved the model's silhouette.

210 The massive amount of geometry present in a forest scene is ${ }_{211}$ not the only difficulty. Realistic lighting is also a challenge at all 212 levels of detail. Behrendt et al. [39] approximated plant models ${ }_{213}$ using billboards generated from a clustering algorithm applied 214 to the model's components. Their technique incorporated real215 istic illumination via spherical harmonics, while approximating 216 distant vegetation with shell textures. Bruneton and Neyret [40] ${ }_{217}$ presented a system that uses two scales based on light fields and 218 height fields to render millions of trees in real-time with realis${ }_{219}$ tic lighting.

\section{3. Crown reconstruction}

\section{3.1. Input photograph assumptions}

${ }_{222}$ Our method starts from a single picture of a tree and a seg${ }_{223}$ mentation mask for its crown foliage area. Analyzing automatic 224 matting algorithms was out of the scope of this work. We inter225 actively generate this segmentation in a few seconds using the 226 segmentation tools described in [41], and applying a contrac227 tion and smoothing afterwards to the segmented contour.

Currently, our approach works best when the following con229 ditions about the input photograph are met: 234 235
- The crown foliage should be dense, the underlying branching structure should be hidden or hardly visible.

- The crown brightness should be influenced by selfocclusions (this is often a result of dense crown foliage).

- The crown shape can be approximated by a single volume. In the future we might extend the method to deal with trees with sparse foliage nuclei.

- The leaf system should not exhibit strong, large-scale directional patterns as in, e.g. palm trees, which are not supported by our approach. Small scale directional patterns (e.g. preferred leaf orientations due to phototropism and high-frequency features alike) have little impact on our synthesized textures and are thus well supported.

- If the crown is not completely visible, the artist can specify a segmentation to be used for the crown shape, together with another segmentation, to be used for texture synthesis, identifying pixels not belonging to the crown. 

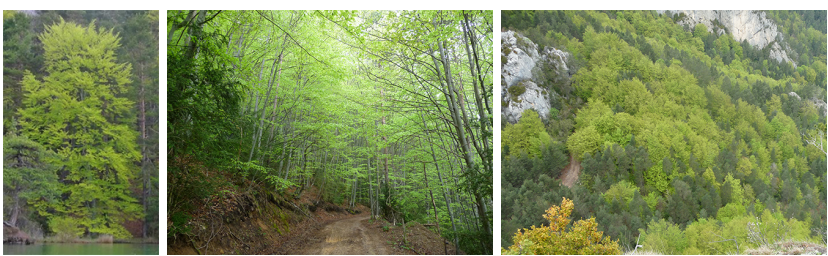

Figure 2: (Left) Individual of Fagus Sylvatica. (Center) Inside a fagus sylvatica forest; the branching structure of close trees is clearly visible. (Right) The same forest seen from about $100 \mathrm{~m}$ above, now with a much more dense and uniform appearance.
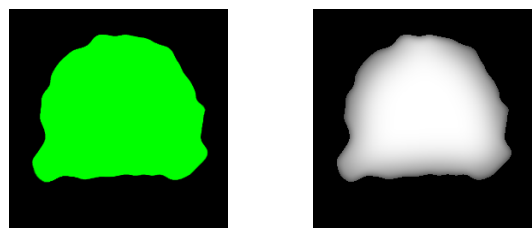

Figure 3: From the tree segmentation (left) we can compute a heightmap that represents the front half of the envelope mesh (right) using the bilaplacian filter.

247 Figure 2 shows one example of tree species (fagus sylvatica) 248 that does not follow our assumptions. It grows a dense crown 249 but its branches are very separated and with a sparse foliage, 250 thus revealing the underlying branching structure. Even for ${ }_{251}$ these cases, their global appearance as seen from afar is dense 252 and uniform, and we can still obtain a plausible reconstruction ${ }_{253}$ suitable for mid-range and distant views.

\section{3.2. Envelope mesh}

255 The first step of the reconstruction consists in converting the 256 silhouette given by the segmentation of the input image into a ${ }_{257}$ rough approximation of the tree crown. We will refer to this vol258 ume as the envelope mesh. Sketch-based systems [42] provide 259 algorithms to inflate a mesh from a simple boundary, but results ${ }_{260}$ with typical tree silhouettes are rather poor (see side views in ${ }_{261}$ Figure 8) as elevation values on spine vertices directly depend ${ }_{262}$ on the distance to the silhouette (to make wide areas fat, and 263 narrow areas thin).

264 Since we cannot assume a high-quality segmentation of the ${ }_{265}$ crown's silhouette, we decided to generate a relatively smooth 266 envelope mesh by generating a heightmap over the segmented ${ }_{267}$ image (see Figure 3). This heightmap will provide the frontal 268 half of the envelope mesh, and its inverted version will provide ${ }_{269}$ the other half. Due to the ill-defined nature of this problem, we 270 fix the silhouette points of the heightmap on the $z=0$ plane $(z$ ${ }_{271}$ represents height) and we ask their tangents to be parallel to the ${ }_{272} z$ axis, while requiring that all points inside the crown reduce 273 their bending energy. We achieve this by minimizing the thin${ }_{274}$ plate energy defined by a biharmonic equation inside the crown ${ }_{275} C$, while restricting the silhouette $S$ to have $z=0$. Written as a 276 continuous optimization problem, we want to solve

$$
\min _{z} \int_{C} \nabla^{4} z, \quad \text { such that } \begin{cases}z \geq 0 & \forall p \in C \backslash S \\ z=0 & \forall p \in S \\ z^{\prime}=f(x, y) & \forall p \in S\end{cases}
$$

277 where $\nabla^{4}$ is the biharmonic operator and $f$ is a positive real 278 function controlling the gradient of the distance field at the sil279 houette points.

The optimization problem above can be written as a linear ${ }_{281}$ system for the $z$ values using the bilaplacian filter [43]. We dis${ }_{282}$ cretize the domain $C$ using our input segmentation as a $2 \mathrm{D}$ grid ${ }_{283}$ discretization. In this discretized domain, tangets are specified 284 as follows. We define the outer silhouette $S_{o}$ of the image as the 285 set of pixels outside $C$ but adjacent to a silhouette pixel. In this 286 setting, for each inner pixel $(i, j)$ :

$$
\begin{array}{ll}
z(i, j) \geq 0 & \text { if }(i, j) \in C \backslash S \\
z(i, j)=0 & \text { if }(i, j) \in S \\
z(i, j)=-f(i, j) & \text { if }(i, j) \in S_{o}
\end{array}
$$

Then, for each unknown $z$ we create the equation obtained 288 from the convolution of its corresponding point with its neigh289 bors, representing the thin-plate energy computation:

$$
\begin{gathered}
\sum_{\substack{-2 \leq d_{i} \leq 2 \\
-2 \leq d_{j} \leq 2}} M_{d_{i}+2, d_{j}+2} \cdot z\left(i+d_{i}, j+d_{j}\right)=0 \\
M=\frac{1}{16}\left[\begin{array}{ccccc}
0 & 0 & 1 & 0 & 0 \\
0 & 2 & -8 & 2 & 0 \\
1 & -8 & 20 & -8 & 1 \\
0 & 2 & -8 & 2 & 0 \\
0 & 0 & 1 & 0 & 0
\end{array}\right]
\end{gathered}
$$

290 This convolution is the result of the application of two suc291 cessive laplacian filters (a well known edge detector image fil292 ter) as the bilaplacian itself is the result of computing the lapla293 cian of a laplacian.

294 Solving the system yields promising results, but there are still 295 some issues (as shown in Figure 4-left). Due to the way we ap296 ply the tangents' constraints using the outer silhouette on the 297 grid, they cannot be defined as we wanted - as vectors point298 ing in the $z$ direction - because there will always be a small 299 displacement in $x$ and $y$ between the outer silhouette and the 300 real one. Therefore, the resulting envelope will never look 301 as smooth as we want. Increasing the negative displacement 302 for pixels in $S_{O}$ alleviates this problem but tangents will never ${ }_{303}$ match with the mirrored ones at the silhouette.

304 In order to properly express tangent constraints we decided 305 to extend the previous linear system to $3 \mathrm{D}$ with unknowns for ${ }_{306} x$ and $y$ as well. Thus, inner vertices $(\in C \backslash S)$ will no longer be ${ }_{307}$ restricted to a grid arrangement. In this new setup, grid vertices 308 have coordinates

$$
\begin{array}{ll}
\left(x_{x}, x_{y}, x_{z}\right) & \text { if }(i, j) \in C \backslash S \\
(i, j, 0) & \text { if }(i, j) \in S \\
\left(i^{\prime}, j^{\prime}, f\left(i^{\prime}, j^{\prime}\right)\right) & \text { if }(i, j) \in S_{o}
\end{array}
$$

309 where $x_{x}, x_{y}$ and $x_{z}$ are the unknowns, and $\left(i^{\prime}, j^{\prime}\right)$ is just the 310 projection of $(i, j) \in S_{o}$ onto the closest silhouette pixel. We 

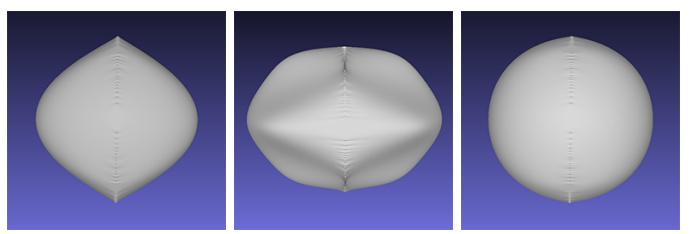

Figure 4: Inflating the envelope while keeping the $x$ and $y$ components fixed does not produce the desired results (left). However, expressing the minimization problem in 3D helps solve this problem (right) but only if a 8-connected silhouette is used. A 4-connected silhouette produces unacceptable artifacts (center).

${ }_{311}$ use Equation 1 independently on each unknown to solve the 312 problem.

${ }_{313}$ Pixel connectivity plays a big role in the reconstruction pro${ }_{314}$ cess. The set of pixels in $S$ contains pixels classified as in315 side the crown but adjacent to pixels outside. If we assume ${ }_{316} 4$-connectivity for the silhouette pixels, results are not suffi${ }_{317}$ ciently smooth (e.g. we cannot reconstruct a sphere from a ${ }_{318}$ circle boundary, as shown in Figure 4). Using 8-connectivity, ${ }_{319}$ however, solves these artifacts.

320 The first columns of Figure 8 show some tree silhouettes and ${ }_{321}$ the resulting crowns based on the envelope meshes. As for ${ }_{322}$ function $f(x, y)$, artists could chose either a constant function ${ }_{323} f(x, y)=k$ (for roughly spherical crowns) or a quadratic func324 tion with the form $f(x, y)=a y^{2}+1$ (for approximately conical 325 trees). In both cases we assume $Y$ is the vertical axis pointing 326 downwards, and $(x, y)$ are normalized pixel coordinates. Next ${ }_{327}$ sections explain how we add color and relief details to these 328 meshes.

\section{3.3. Color synthesis}

3зо A single picture of a tree contains less than a half of the tree's ${ }_{331}$ outer surface. However, it is reasonable to assume that the oc${ }_{332}$ cluded parts will have a similar appearance than those visible. ${ }_{333}$ We thus use a texture synthesis approach to recover missing 334 parts.

${ }_{335}$ First, the segmented photograph is scaled to have unit aspect ${ }_{336}$ ratio, and placed at the center of a 4:3 canvas such that it occu${ }_{337}$ pies $5 / 12$ of its width and $5 / 9$ of its height. We do this under the 338 assumption that the original photograph must cover a face of ${ }_{3 з 9}$ the output cubemap plus about a third of the neighboring faces. ${ }_{340}$ We then fill the rest of the canvas using the texture synthesis ${ }_{341}$ algorithm from [44].

${ }_{342}$ The color cubemap is constructed by cropping the six faces ${ }_{343}$ of this synthetic texture, as shown in Figure 5, and applying ${ }_{344}$ a local synthesis again on the bands around the borders of the ${ }_{345}$ faces that were not originally in contact. This additional step ${ }_{346}$ guarantees continuity across all cubemap edges.

\section{3.4. Relief synthesis}

348 Our relief estimation method is inspired by shape-from349 shading (SFS) algorithms like [45]. Unfortunately, we can as${ }_{350}$ sume very little about the lighting conditions of the input pho${ }_{351}$ tographs, and typical assumptions in SFS approaches (known ${ }_{352}$ reflectance model, known direction of light sources) do not

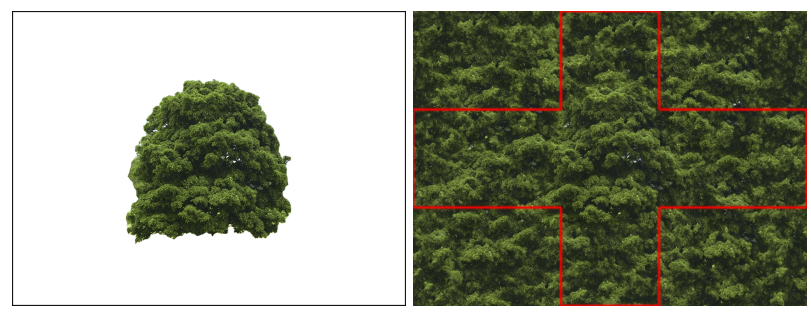

Figure 5: Left: placement of the segmented photograph inside the canvas to be synthesized. Right: synthesis result and cubemap outline. Notice that the synthesized result includes the segmented photograph.

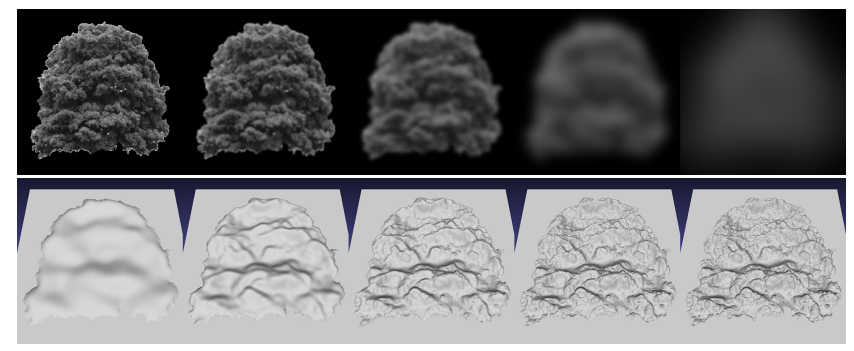

Figure 6: Gaussian decomposition pyramid (top) and multiscale relief generation (bottom). Each heightfield is the result of adding its corresponding Gaussian pyramid level to the previous one, and decreasing the weight at each step.

${ }_{353}$ hold in our case. Furthermore, tree leaves form complex struc354 tures with high-frequency variation across the image, which is 355 a worst-case scenario for SFS approaches, which describe the ${ }_{356}$ surface shape either in terms of the surface normal, or in terms 357 of the heightfield gradient.

${ }_{358}$ Since we assume the envelope mesh already captures the 359 rough shape of the crown, we directly estimate depth from lu360 minance. Despite the fact that a tree has a huge number of self${ }_{361}$ occlusions from the different branches and leaves, a fraction of 362 incoming light is likely to reach occluded portions due to light ${ }_{363}$ scattering and non-opaque leaves. The deeper we are inside the ${ }_{364}$ crown, the darker we expect it to be. Again we assume a dense 365 input crown, preventing deep areas close to the crown center 366 from receiving direct light.

367 We start by computing the luminance of the input image and ${ }_{368}$ normalizing it to be in the $[0,1]$ range. Then, we compute a 369 blur pyramid of this luminance image, similar to the Gaussian 370 pyramid of Burt and Adelson [46]. We also tried a Laplacian ${ }_{371}$ decomposition, but the relief obtained from the Gaussian is 372 more prominent due to lower frequencies being counted mul373 tiple times.

374 Once we have $N$ levels of the pyramid (we tested $N=4$ and ${ }_{375} N=5$ ), we combine them using an exponentially-decreasing set 376 of weights. Lower levels (blurrier) will have larger weights be377 cause they represent large-scale (and potentially deeper) struc378 tures, while higher levels are usually noisier and just add small 379 fine details to the relief.

Figure 6 shows an example of a relief reconstruction; the fig${ }_{381}$ ure uses the input tree image for clarity; we extract the relief ${ }_{382}$ from the synthetic cube map described above. 

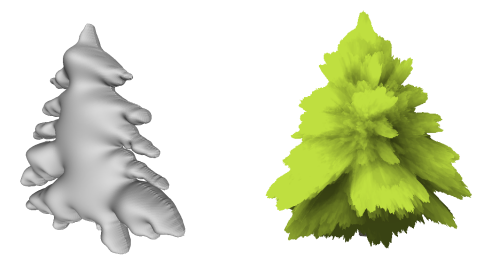

Figure 7: Base envelope mesh computed from a segmentation (left). Tree crown created from three rotated copies of the envelope and relief perturbation (right).
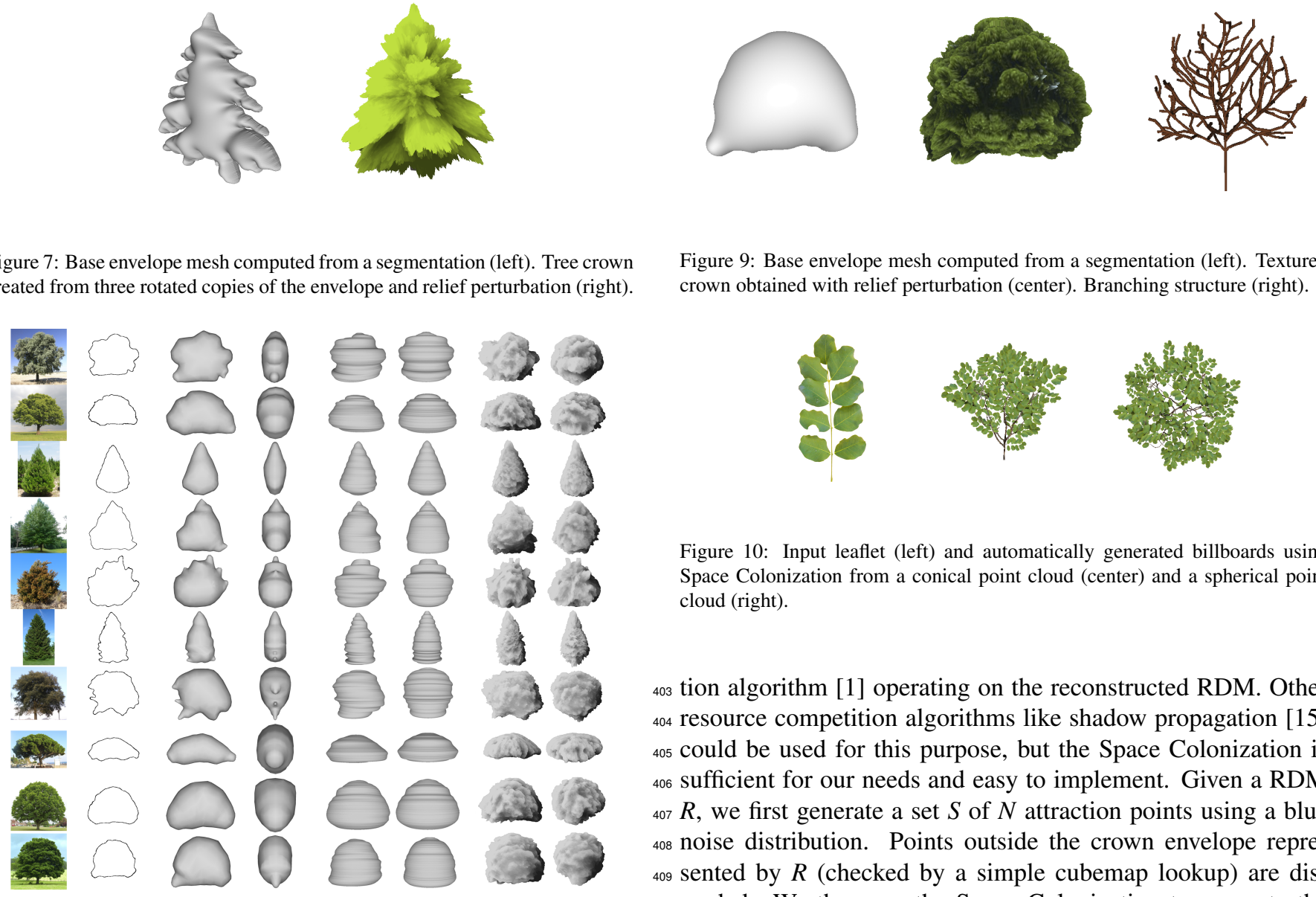

Figure 9: Base envelope mesh computed from a segmentation (left). Textured crown obtained with relief perturbation (center). Branching structure (right).
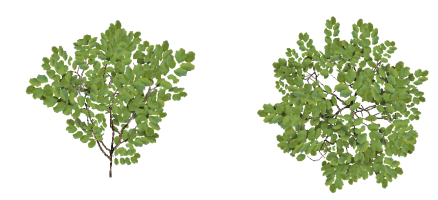

Figure 10: Input leaflet (left) and automatically generated billboards using Space Colonization from a conical point cloud (center) and a spherical point cloud (right).

Figure 8: From left to right: input photograph, segmented silhouette, front and side views from a sketch-based system [42], front and side views using a function-based crown model from [36], and front and side views of our reconstructed crowns.

\section{3вз 3.5. Radial distance map}

384 The final representation of the crown shape is the radial dis385 tance map (RDM). For each direction, we store the distance 386 from the crown center to the crown surface. This representation ${ }_{387}$ is the result of perturbing the crown envelope with the relief 388 values extracted from the color cubemap. At build time, we звя just render the envelope mesh and compute the final radius on ${ }_{390}$ a fragment shader as $\|p-c\| \cdot(1+\rho \cdot(h(p-c)-0.5))$, where $p$ 391 is a point on the envelope surface, $c$ is the center of the crown, ${ }_{392} h(p-c)$ is the relief cubemap value in the direction $p-c$, and ${ }_{393} \rho$ is a parameter that controls the weight of the relief map. In ${ }_{394}$ order to improve the overall shape of certain types of trees, the 395 artist may choose to add several rotated copies of the crown ${ }_{396}$ envelope (as an example see Figure 7). This just involves ren${ }_{397}$ dering the envelope mesh multiple times and keeping the max398 imum radius value (through depth test) in the output cubemap.

399 Figures 8 and 16 show some reconstructed volumes.

\section{3.6. Branches and leaves}

${ }_{401}$ In order to support highly detailed models, a branching struc${ }_{402}$ ture for the crown can be generated with the Space Coloniza-

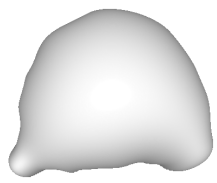

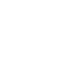

403 tion algorithm [1] operating on the reconstructed RDM. Other 404 resource competition algorithms like shadow propagation [15] 405 could be used for this purpose, but the Space Colonization is 406 sufficient for our needs and easy to implement. Given a RDM ${ }_{407} R$, we first generate a set $S$ of $N$ attraction points using a blue 408 noise distribution. Points outside the crown envelope repre409 sented by $R$ (checked by a simple cubemap lookup) are dis${ }_{410}$ carded. We then run the Space Colonization to generate the 411 branching structure. Figure 9 shows an example crown and the 412 branching structure obtained. Note that only the main branches ${ }_{413}$ are represented.

${ }_{414}$ In order to keep real time framerates during rendering, we ${ }_{415}$ use billboards to represent groups of leaves and small branches. ${ }_{416}$ These billboards are created by instantiating multiple copies of 417 a leaflet (a photograph of leaves or small branches, or even a 3D ${ }_{418}$ mesh if available). The distribution and arrangement of leaflets ${ }_{419}$ inside each billboard is also based on the Space Colonization ${ }_{420}$ algorithm with an arbitrary input volume. We found that using ${ }_{421}$ simple shapes like spheres or cones yields convincing results ${ }_{422}$ (see Figure 10). Conical volumes yield billboards representing ${ }_{423}$ branchlets that will be connected from the base of the cone to ${ }_{424}$ the branching structure. Spherical clouds, although less real425 istic when seen in isolation, can be rotated arbitrarily around ${ }_{426}$ the connecting point - their center - and thus can be oriented at ${ }_{427}$ runtime to face the viewer at any time.

${ }_{428}$ Fully-detailed tree models can also be generated by increas${ }_{429}$ ing the refinement of the branching structure. Figure 11 shows a ${ }_{430}$ couple of tree models generated using this approach in about 90 ${ }_{431}$ seconds (Python code running on Blender). Notice that the final ${ }_{432}$ shape of the tree models closely matches that of the crown en${ }_{433}$ velopes. In the examples, we used (in [1] notation) $N=10,000$ ${ }_{434}$ attraction points (to ensure a dense distribution of points within ${ }_{435}$ the crown), a kill distance $d_{k}=0.01 r$ ( $r$ being the average ra436 dius of the crown envelope) to ensure that the branching system ${ }_{437}$ provides a dense coverage of the crown volume, and an intern- 

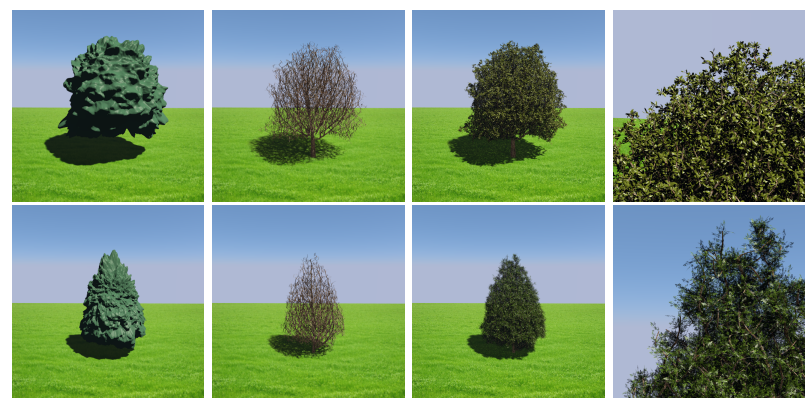

Figure 11: Fully-detailed models created by generating attraction points within the crown envelope and then using a space colonization algorithm. The images show the crown envelope, the branching system, and the final tree (full/detail).

${ }_{438}$ ode length $D=0.5 k_{d}$. The resulting models have around $300 \mathrm{~K}$ ${ }_{439}$ textured quads (leaves) plus about $100 \mathrm{~K}$ quads (branching sys${ }_{440}$ tem) and their use should be reserved to very close-up views.

\section{${ }_{441}$ 4. Crown rendering}

${ }_{442}$ The algorithms above construct a radial distance map and a ${ }_{443}$ color map (both represented as cube maps) for each tree photo${ }_{444}$ graph given as input. This representation can be rendered with ${ }_{445}$ increasing levels of detail, as described below.

\section{${ }_{446}$ 4.1. Direct rendering}

447 Our first rendering method implements a direct visualization ${ }_{448}$ of the RDM through fragment-based relief mapping. Relief ${ }_{449}$ mapping approaches [47] cannot compete with current tessel450 lation engines when close-up views of detailed geometry are ${ }_{451}$ required, but offer excellent performance for distant objects (es${ }_{452}$ pecially in deferred shading setups) since their rendering cost is ${ }_{453}$ output-sensitive, i.e. the cost directly depends on the number of ${ }_{454}$ covered pixels [48]. This is thus the LoD we use for rendering 455 distant trees.

${ }_{456}$ The classic approach for relief mapping [47] is to compute 457 the ray-heightfield intersection by sampling points along the ${ }_{458}$ ray, first using linear search to find a sample inside the object, 459 and then refining the intersection point through binary search. ${ }_{460}$ For each sampled point, the height of the sample is compared ${ }_{461}$ with the depth stored in the relief map to determine whether ${ }_{462}$ the sample is inside or outside the heightfield. We apply this ${ }_{463}$ idea to radial distance maps. When traversing the ray along 464 the viewing direction, ray samples are computed in world space ${ }_{465}$ (instead of tangent space). For a ray sample $P$, we compute ${ }_{466} \vec{v}=(P-C) / R_{\max }\left(C\right.$ is the crown center and $R_{\max }$ is the maxi${ }_{467}$ mum crown radius), and check if $P$ is inside the crown by com${ }_{468}$ paring $\|\vec{v}\|$ with the radial distance value stored for direction $\vec{v}$. ${ }_{469}$ We considered two options for computing the fragment color. ${ }_{470}$ The simplest option is to get the color directly from the color ${ }_{471}$ cube map. However, crown parts along the same radial direc${ }_{472}$ tion get the same color from the cube map, resulting in some ${ }_{473}$ texture stretching that might be noticeable in trees with promi${ }_{474}$ nent branches and abrupt relief changes. Alternatively, we can ${ }_{475}$ get the fragment color from the central part of the synthetic $2 \mathrm{D}$

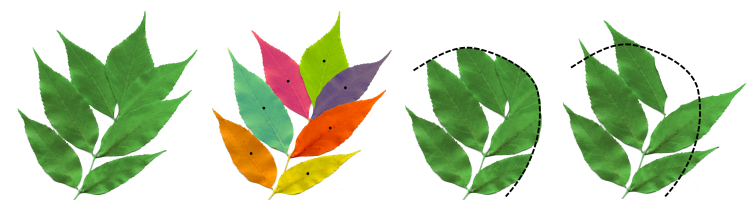

Figure 12: Rendering clipped billboard clouds: (a) leaf texture; (b) segmentation showing individual leaf centroids, (c) per-fragment clipping, (d) per-leaf clipping, based on the inside/outside classification of the leaf centroid.

476 texture (Figure 5), using the same texture coordinates we would 477 use for a planar billboard. This second option provides higher 478 quality images, but the re-usage of the same texture portion for 479 all view directions becomes apparent when rotating the camera 480 around a closep-up tree. We thus use this second option only ${ }_{481}$ for distant trees. Notice that with both options the rendered tree ${ }_{482}$ silhouette matches that of the RDM.

\section{${ }_{483}$ 4.2. Clipped billboard clouds}

${ }_{484}$ For closer views we add high-frequency details by drawing 485 a billboard cloud with an RGBA texture showing leaves and ${ }_{486}$ small branches. Billboard centers are placed on points of the ${ }_{487}$ branching structure generated using the Space Colonization al${ }_{488}$ gorithm (see Section 3.6). A single vertex buffer is shared 489 among all tree instances of the same species. The actual num490 ber of points to render (converted into quads in the geometry ${ }_{491}$ shader) varies for each instance, depending on the distance to ${ }_{492}$ the viewer, as in [37].

493 In order to preserve the overall crown shape of the species, 494 we clip the geometry outside the crown volume. Again, a point${ }_{495}$ inside-crown test requires a single texture lookup to the RDM. ${ }_{496}$ This clipping operation can be performed at different granular${ }_{497}$ ity levels. Discarding complete billboards based on their center 498 (e.g. in the geometry shader) is the simplest option, but tends to ${ }_{499}$ produce popping artifacts around the tree silhouette when large ${ }_{500}$ textures are used. Discarding individual fragments faithfully ${ }_{501}$ preserves the crown shape (except for transparent texels in the 502 leaf texture), but tends to cut individual leaves in the texture. A ${ }_{503}$ better option is to discard fragments based on the centroid of the 504 individual leaves in the texture (Figure 12). This requires addi505 tional segmentation information where individual leaves point ${ }_{506}$ towards the cell centroid. We automatically generate this in${ }_{507}$ formation when we create the billboards. This technique yields 508 better silhouettes at the only expense of an extra texture lookup. 509 Apart from the leaf centroid, we also store other information 510 in the billboards (see Figure 13). Normals and precomputed 511 ambient occlusion are used during shading. Depth is used to ${ }_{512}$ reduce the artifacts when two billboards cross. The type bit ${ }_{513}$ (shown in black and gray for clarity) separates the leaves from 514 the branches, and we use this information during specular light515 ing. Finally, the texture coordinates refer to the coordinates 516 used to instantiate the original photograph (see Figure 10). 

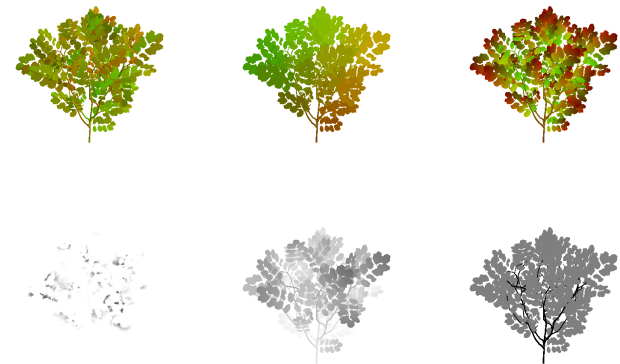

Figure 13: Information stored for the generated leaves billboards besides the RGBA components. Left to right and top to bottom: normals, leaf centroids, texture coordinates, ambient occlusion, depth, type bit.
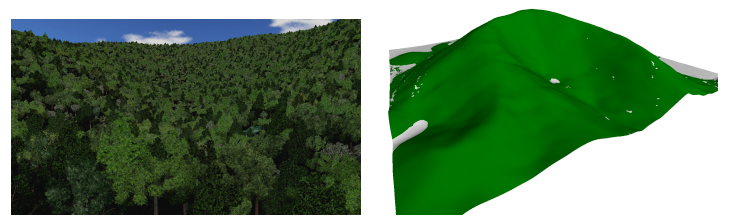

Figure 14: Test scene used for performance analysis. The image on the right shows tree areas.

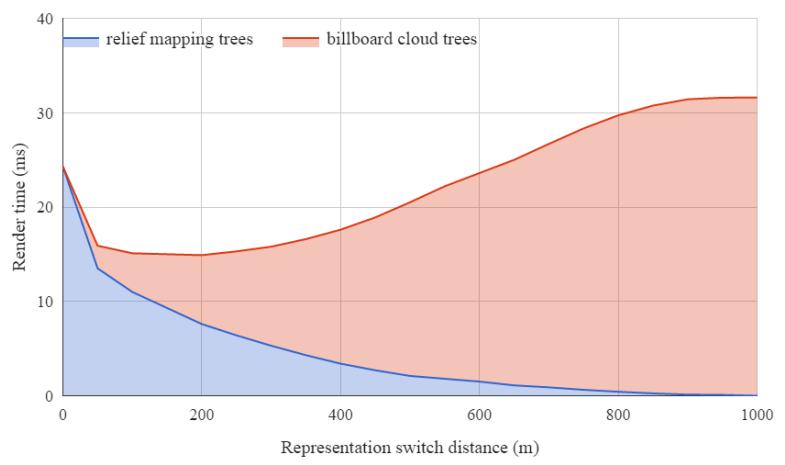

Figure 15: Accumulated rendering times of both LoD representations depending on the switch distance.

\section{5. Results}

\section{${ }_{518}$ 5.1. Rendering performance}

${ }_{519}$ We measured the rendering performance of our tree repre520 sentations on a dense forest scene (Figure 14). Accurate se${ }_{521}$ lection and placement of appropriate trees for a given terrain is ${ }_{522}$ out of the scope of this paper (see e.g. [49] for segmentation of ${ }_{523}$ aerial images, and [36] for modulating the appearance of tree 524 instances with color from aerial images). We thus randomly ${ }_{525}$ placed $50 \mathrm{~K}$ trees in green areas of the aerial image, following ${ }_{526}$ a blue noise distribution. Tree instances had varying sizes. We ${ }_{527}$ chose a view with a large number of visible trees (42K trees, ${ }_{528}$ Figure 14-left) with a broad range of distances (up to about $5291,000 \mathrm{~m})$.

${ }_{530}$ Rendering times were measured with different distance ${ }_{531}$ thresholds $(d)$ for the LoD switch. Figure 15 shows average ren-
${ }_{532}$ dering times on NVIDIA GTX 970 hardware on a high-quality 53з setting: Full HD $(1920 \times 1080)$ resolution and $8 \times$ multisam${ }_{534}$ pling. All tree instances within $d$ distance were rendered using ${ }_{535}$ the detailed (billboard cloud) representation, using a varying ${ }_{536}$ number of billboards per instance (from 16 to 144) according ${ }_{537}$ to the screen-projected area. Tree instances beyond $d$ were ren${ }_{538}$ dered using the direct (relief-mapping) representation.

${ }_{539}$ For the detailed representation, the throughput varied from ${ }_{540} 700$ trees/ms (only the closest trees being rendered with the de${ }_{541}$ tailed representation) to 1,300 trees/ms (all trees rendered as 542 billboard clouds). For the direct rendering, the throughput var${ }_{543}$ ied from 1,700 trees/ms (all trees rendered through relief map${ }_{544} \mathrm{ping}$ ) to 12,000 trees $/ \mathrm{ms}$ (only distant trees rendered with relief 545 mapping). For distant trees, the relief mapping was up to one 546 order of magnitude faster than billboard clouds, mainly due to 547 its output sensitive nature.

548 From a visual quality point of view, we found out that the ${ }_{549}$ distance threshold $d$ should be greater that $150 \mathrm{~m}$ (for smaller 550 values the artifacts of the relief representation become too ap${ }_{551}$ parent). From a performance point of view, optimal $d$ values 552 were about $200 \mathrm{~m}$ (due to the relief map representation to be${ }_{553}$ come inefficient for tree instances covering a large part of the 554 viewport, whereas the per-instance cost for the detailed repre555 sentation remains roughly constant once they reach the mini556 mum number of billboards). Frame rates for $d=200 \mathrm{~m}$ were ${ }_{557}$ above $60 \mathrm{fps}$ even for this dense forest area.

\section{5.2. Memory footprint}

559 For each tree species we need to store the following data: a ${ }_{560}$ RDM encoded as a single-channel cubemap $(6 \times 256 \times 256)$, a ${ }_{561}$ RGB color texture $(1024 \times 768)$, a small number of billboard 562 textures $(512 \times 512$, encoding RGBA channels plus 8 bytes per ${ }_{563}$ pixel for the additional information), and a branching structure ${ }_{564}$ (about 200-400 3D points). Note that with only the RDM and 565 the color texture (4.5 MB of uncompressed data) we can already 566 render trees with the direct representations. For the detailed ${ }_{567}$ representation we used four billboard textures per species, each 568 texture taking $3 \mathrm{MB}$ of uncompressed data. The total memory ${ }_{569}$ footprint per species is less than $17 \mathrm{MB}$. Using standard com570 pressed texture formats, the footprint per species is about $5 \mathrm{MB}$, 571 which is negligible when compared with aerial imagery.

\section{5.3. Tree reconstruction}

573 The results of our crown reconstruction algorithm with var574 ious tree photographs are shown in Figure 16. Notice that the 575 overall crown shape and appearance are preserved. The average 576 construction time (on an Intel Core i7 CPU) including manual 577 intervention was $5 \mathrm{~min}$. User segmentation of the crown shape 578 in the photograph took less than a minute in an interactive edi${ }_{579}$ tor. The most expensive step (about 1-2 min) was the synthesis 580 of the color texture (Section 3.3). In our tests, we generated 581 a $1024 \times 768$ image. The rest of the reconstruction steps, in582 cluding solving the bilaplacian linear system, the computation ${ }_{583}$ of the Gaussian pyramid and the construction of the RDM took 584 less than $5 \mathrm{~s}$. Space Colonization to produce the main branching ${ }_{585}$ structure and each billboard run in less than a second. Since we 


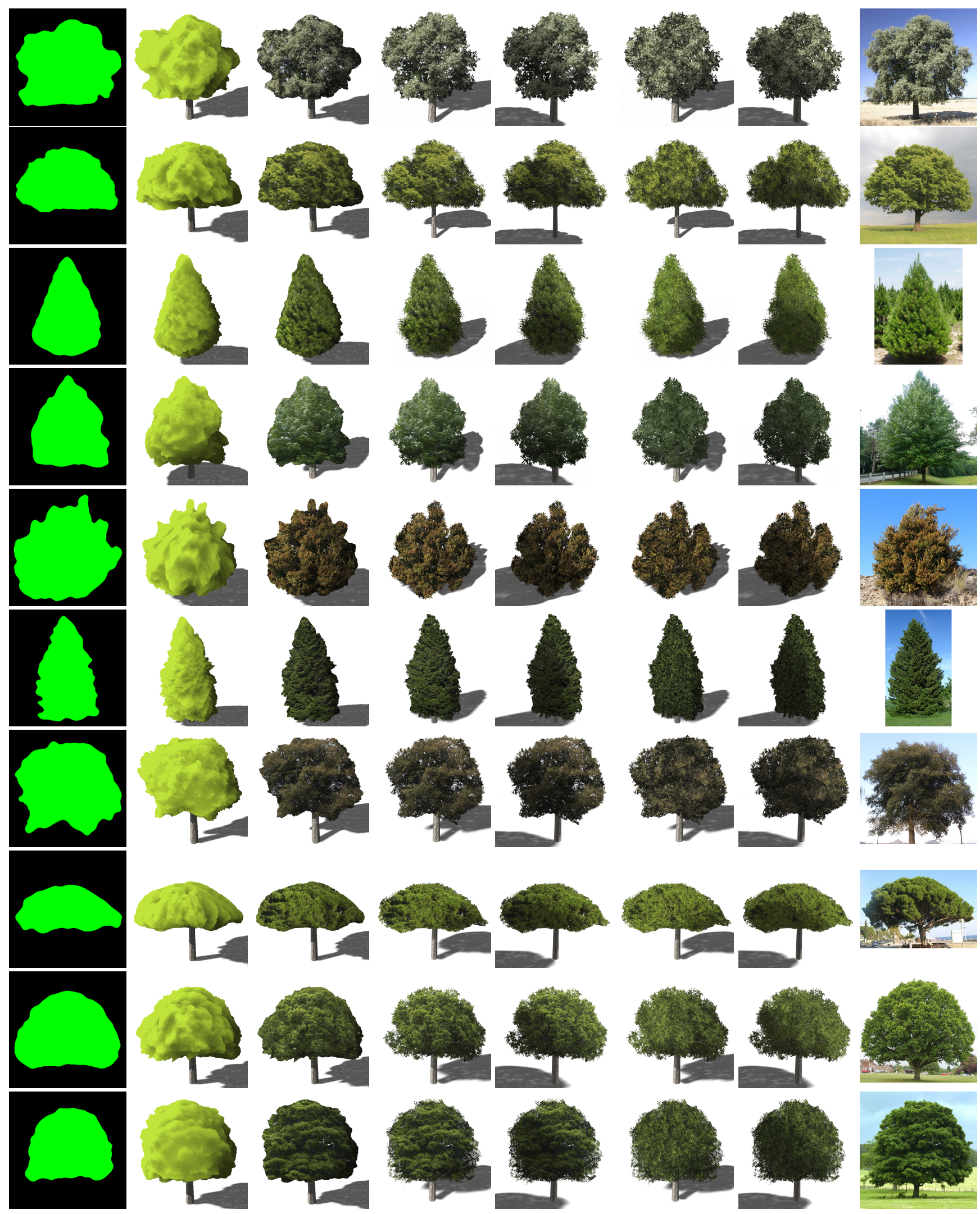

Figure 16: Examples of trees reconstructed using our method. From left to right: segmentation mask, front view of the volume represented by the radial distance map, the same view using the color texture, two lightings of the billboard cloud textured from the color texture, two lightings of the billboard cloud using the color from the billboards, and the original photograph. 
${ }_{586}$ only wanted the overall structure, we used only a few thousand 587 attractors.

\section{${ }_{588}$ 6. Conclusions}

${ }_{589}$ In this paper we have proposed a pipeline for helping artists 590 to create plausible tree models from individual photographs 591 with minimal authoring efforts. The resulting models can be 592 used to synthesize local vegetation on top of DTMs represent${ }_{593}$ ing real scenarios. The fact that a single tree photograph suf594 fices allows artists to benefit from available georeferenced pho595 tographs to populate arbitrary forest areas. Unlike compet596 ing approaches, reconstructed trees are encoded in a compact, 597 image-based, easy to compress representation, suitable for net598 work streaming, and support efficient rending from arbitrary 599 view directions.

600 Some assumptions (Section 3) are needed for our approach 601 to work. Essentially, our method works with trees with dense, 602 single-nucleus foliage without large-scale directional features. 603 Interesting avenues for future work include the support of 604 sparse crowns and multiple-nuclei foliage, the computation and 605 use of opacity values from the input image, and automatic ex606 traction of leaf patches for the billboard textures. Our approach 607 would greatly benefit from advances in automatic segmentation 608 of tree crowns in photographs, as well as improvements in au609 tomatic recognition of vegetation in aerial images.

\section{${ }_{610}$ References}

1] A. Runions, B. Lane, P. Prusinkiewicz, Modeling Trees with a Space Colonization Algorithm, in: Proceedings of the Third Eurographics Conference on Natural Phenomena, NPH'07, Eurographics Association, Airela-Ville, Switzerland, Switzerland, 2007, pp. 63-70.

[2] H. Honda, Description of the form of trees by the parameters of the treelike body: Effects of the branching angle and the branch length on the shape of the tree-like body, Journal of Theoretical Biology 31 (2) (1971) $331-338$.

[3] M. Aono, T. Kunii, Botanical tree image generation, IEEE Comput. Graph. Appl. 4 (5) (1984) 10-34.

[4] P. E. Oppenheimer, Real time design and animation of fractal plants and trees, in: Proc. of SIGGRAPH '86, ACM, 1986, pp. 55-64.

[5] J. Weber, J. Penn, Creation and rendering of realistic trees, in: Proceedings of the $22 \mathrm{Nd}$ Annual Conference on Computer Graphics and Interactive Techniques, SIGGRAPH '95, ACM, New York, NY, USA, 1995, pp. 119-128.

[6] O. Stava, S. Pirk, J. Kratt, B. Chen, R. Měch, O. Deussen, B. Benes, Inverse procedural modeling of trees, Computer Graphics Forum 33 (6) (2014) 118-131.

[7] J. Bloomenthal, Modeling the mighty maple, SIGGRAPH Comput. Graph. 19 (3) (1985) 305-311.

[8] A. Lindenmayer, Mathematical models for cellular interactions in development: Parts i and ii, Journal of theoretical biology 18 (3) (1968) 280315.

[9] P. Prusinkiewicz, M. S. Hammel, E. Mjolsness, Animation of plant development, in: Proceedings of the 20th Annual Conference on Computer Graphics and Interactive Techniques, SIGGRAPH '93, ACM, New York, NY, USA, 1993, pp. 351-360.

39 [10] R. Měch, P. Prusinkiewicz, Visual models of plants interacting with their environment, in: Proceedings of the 23rd Annual Conference on Computer Graphics and Interactive Techniques, SIGGRAPH '96, ACM, New York, NY, USA, 1996, pp. 397-410.

643 [11] P. Prusinkiewicz, L. Mündermann, R. Karwowski, B. Lane, The use of po644 sitional information in the modeling of plants, in: Proceedings of the 28th
Annual Conference on Computer Graphics and Interactive Techniques, SIGGRAPH '01, ACM, New York, NY, USA, 2001, pp. 289-300.

7 [12] P. Prusinkiewicz, A. Lindenmayer, The Algorithmic Beauty of Plants, Springer-Verlag New York, Inc., New York, NY, USA, 1996.

49 [13] J. O. Talton, Y. Lou, S. Lesser, J. Duke, R. Měch, V. Koltun, Metropolis procedural modeling, ACM Trans. Graph. 30 (2) (2011) 11:1-11:14.

51 [14] B. Lintermann, O. Deussen, Interactive modeling of plants, IEEE Comput. Graph. Appl. 19 (1) (1999) 56-65.

53 [15] W. Palubicki, K. Horel, S. Longay, A. Runions, B. Lane, R. Měch, P. Prusinkiewicz, Self-organizing tree models for image synthesis, in: ACM SIGGRAPH 2009 Papers, SIGGRAPH '09, ACM, New York, NY, USA, 2009, pp. 58:1-58:10.

[16] S. Longay, A. Runions, F. Boudon, P. Prusinkiewicz, TreeSketch: Interactive Procedural Modeling of Trees on a Tablet, in: K. Singh, L. B. Kara (Eds.), Eurographics Workshop on Sketch-Based Interfaces and Modeling, The Eurographics Association, 2012.

[17] S. Pirk, O. Stava, J. Kratt, M. A. M. Said, B. Neubert, R. Měch, B. Benes, O. Deussen, Plastic trees: Interactive self-adapting botanical tree models, ACM Trans. Graph. 31 (4) (2012) 50:1-50:10.

[18] S. Pirk, T. Niese, T. Hädrich, B. Benes, O. Deussen, Windy trees: Computing stress response for developmental tree models, ACM Trans. Graph. 33 (6) (2014) 204:1-204:11.

[19] I. Shlyakhter, M. Rozenoer, J. Dorsey, S. Teller, Reconstructing 3D Tree Models from Instrumented Photographs, IEEE Computer Graphics and Applications 21 (3) (2001) 53-61.

[20] A. Reche, I. Martin, G. Drettakis, Volumetric Reconstruction and Interactive Rendering of Trees from Photographs, ACM Transactions on Graphics 23 (3) (2004) 720-727.

[21] B. Neubert, T. Franken, O. Deussen, Approximate Image-based Treemodeling Using Particle Flows, ACM Trans. Graph. 26 (3).

[22] L. Quan, P. Tan, G. Zeng, L. Yuan, J. Wang, S. B. Kang, Image-based Plant Modeling, ACM Trans. Graph. 25 (3) (2006) 599-604.

677 [23] P. Tan, G. Zeng, J. Wang, S. B. Kang, L. Quan, Image-based Tree Modeling, ACM Transactions on Graphics 26 (3) (2007) 87.

72 [24] D. Bradley, D. Nowrouzezahrai, P. Beardsley, Image-based Reconstruction and Synthesis of Dense Foliage, ACM Trans. Graph. 32 (4) (2013) 74:1-74:10.

2 [25] H. Xu, N. Gossett, B. Chen, Knowledge and heuristic-based modeling of laser-scanned trees, ACM Trans. Graph. 26 (4).

84 [26] Y. Livny, F. Yan, M. Olson, B. Chen, H. Zhang, J. El-Sana, Automatic Reconstruction of Tree Skeletal Structures from Point Clouds, ACM Transactions on Graphics 29 (6) (2010) 151:1-151:8.

7 [27] Y. Livny, S. Pirk, Z. Cheng, F. Yan, O. Deussen, D. Cohen-Or, B. Chen, Texture-lobes for Tree Modelling, ACM Trans. Graph. 30 (4) (2011) 53:1-53:10.

[28] M. Okabe, S. Owada, T. Igarashi, Interactive Design of Botanical Trees using Freehand Sketches and Example-based Editing, Computer Graphics Forum 24 (3) (2005) 487-496.

[29] J. Wither, F. Boudon, M.-P. Cani, C. Godin, Structure from silhouettes: a new paradigm for fast sketch-based design of trees, Computer Graphics Forum 28 (2) (2009) 541-550.

6 [30] P. Tan, T. Fang, J. Xiao, P. Zhao, L. Quan, Single Image Tree Modeling, ACM Transactions on Graphics 27 (5) (2008) 108:1-108:7.

698 [31] F. Boudon, A. Meyer, C. Godin, Survey on Computer Representations of Trees for Realistic and Efficient Rendering, Rapport de recherche 2301, LIRIS, Université Claude Bernard Lyon 1 (2006).

701 [32] W. T. Reeves, R. Blau, Approximate and probabilistic algorithms for shading and rendering structured particle systems, SIGGRAPH Comput. Graph. 19 (3) (1985) 313-322.

[33] O. Deussen, C. Colditz, M. Stamminger, G. Drettakis, Interactive visualization of complex plant ecosystems, in: Proceedings of the Conference on Visualization '02, VIS '02, IEEE Computer Society, Washington, DC, USA, 2002, pp. 219-226.

[34] G. Gilet, A. Meyer, F. Neyret, Point-based rendering of trees, in: Proceedings of the First Eurographics Conference on Natural Phenomena, 2005.

[35] P. Decaudin, F. Neyret, Rendering forest scenes in real-time., in: A. Keller, H. W. Jensen (Eds.), Rendering Techniques, Eurographics Association, 2004, pp. 93-102.

4 [36] C. Andújar, A. Chica, M. A. Vico, S. Moya, P. Brunet, Inexpensive reconstruction and rendering of realistic roadside landscapes, Computer Graph- 
ics Forum 33 (6) (2014) 101-117.

717 [37] R. L. Cook, J. Halstead, M. Planck, D. Ryu, Stochastic simplification of aggregate detail, in: ACM SIGGRAPH 2007 Papers, SIGGRAPH '07, ACM, New York, NY, USA, 2007.

[38] B. Neubert, S. Pirk, O. Deussen, C. Dachsbacher, Improved model- and view-dependent pruning of large botanical scenes, Computer Graphics Forum 30 (6) (2011) 1708-1718.

[39] S. Behrendt, C. Colditz, O. Franzke, J. Kopf, O. Deussen, Realistic realtime rendering of landscapes using billboard clouds, Computer Graphics Forum (Proceedings of EUROGRAPHICS 2005), 24(3).

[40] E. Bruneton, F. Neyret, Real-time Realistic Rendering and Lighting of Forests, Computer Graphics Forum 31 (2pt1) (2012) 373-382.

728 [41] G. Friedland, K. Jantz, R. Rojas, Siox: simple interactive object extraction in still images, in: Multimedia, Seventh IEEE International Symposium on, 2005, pp. 7 pp.-.

[42] T. Igarashi, S. Matsuoka, H. Tanaka, Teddy: a sketching interface for 3d freeform design, in: ACM SIGGRAPH 1999, ACM, 1999, pp. 409-416.

733 [43] L. Kobbelt, S. Campagna, J. Vorsatz, H.-P. Seidel, Interactive multiresolution modeling on arbitrary meshes, in: Proceedings of the 25th Annual Conference on Computer Graphics and Interactive Techniques, SIGGRAPH '98, 1998, pp. 105-114.

[44] P. Harrison, Image texture tools, Ph.D. thesis, PhD thesis, Monash University (2005).

${ }_{39}$ [45] M. Glencross, G. J. Ward, F. Melendez, C. Jay, J. Liu, R. Hubbold, A perceptually validated model for surface depth hallucination, in: ACM SIGGRAPH 2008 Papers, ACM, 2008, pp. 59:1-59:8.

[46] P. J. Burt, E. H. Adelson, The laplacian pyramid as a compact image code, IEEE Transactions on Communications 31 (1983) 532-540.

74 [47] F. Policarpo, M. M. Oliveira, J. L. D. Comba, Real-time relief mapping on arbitrary polygonal surfaces, in: Proceedings of the 2005 Symposium on Interactive 3D Graphics and Games, I3D '05, ACM, New York, NY, USA, 2005, pp. 155-162.

[48] A. Beacco, N. Pelechano, C. Andujar, A survey of real-time crowd rendering, Computer Graphics Forum Article first published online: 15 Oct 2015. doi:10.1111/cgf.12774.

[49] L. Ruiz, A. Fdez-Sarría, J. Recio, Texture feature extraction for classification of remote sensing data using wavelet decomposition: a comparative study, in: 20th ISPRS Congress, Vol. 35, 2004, pp. 1109-1114. 